\title{
First demonstration of orange-yellow light emitter devices in InGaP/InAIGaP laser structure using strain-induced quantum well intermixing technique
}

\author{
Mohammed A. Majid ${ }^{\mathrm{a}}$, Ahmad A. Al-Jabr ${ }^{\mathrm{a}}$, Rami T. Elafandy ${ }^{\mathrm{a}}$, Hassan M. Oubei ${ }^{\mathrm{a}}$, Mohd S. Alias ${ }^{\mathrm{a}}$, \\ Bayan A. Alnahhas ${ }^{b}$, Dalaver H. Anjum ${ }^{c}$, Tien Khee. $\mathrm{Ng}^{\mathrm{a}}$, Mohamed. Shehata ${ }^{\mathrm{b}}$ and Boon S. Ooi ${ }^{{ }^{\mathrm{a}}}$ \\ ${ }^{a}$ Photonics Laboratory, King Abdullah University of Science \& Technology (KAUST), \\ ${ }^{\mathrm{b}}$ Electrical and Computer Engineering department, Effat University, Jeddah 21478, (KSA) \\ ${ }^{\mathrm{c} A d v a n c e d}$ Nanofabrication, Imaging and Characterization Core Facilities, (KAUST) \\ Thuwal 23955-6900, Kingdom of Saudi Arabia (KSA)
}

\begin{abstract}
In this paper, a novel strain-induced quantum well intermixing (QWI) technique is employed on InGaP/InAlGaP material system to promote interdiffusion via application of a thick-dielectric encapsulant layer, in conjunction with cycle annealing at elevated temperature. Broad area devices fabricated from this novel cost-effective QWI technique lased at room-temperature at a wavelength as short as $608 \mathrm{~nm}$ with a total output power of $\sim 46 \mathrm{~mW}$. This is the shortestwavelength electrically pumped visible semiconductor laser, and the first report of lasing action yet reported from postgrowth interdiffused process. Furthermore, we also demonstrate the first yellow superluminescent diode (SLD) at a wavelength of $583 \mathrm{~nm}$ with a total two-facet output power of $\sim 4.5 \mathrm{~mW}$ - the highest optical power ever reported at this wavelength in this material system. The demonstration of the yellow SLD without complicated multiquantum barriers to suppress the carrier overflow will have a great impact in realizing the yellow laser diode.
\end{abstract}

Keywords: InGaP/InAlGaP, orange \& yellow emitters, lasers, strain, quantum well intermixing, cycle annealing

\section{INTRODUCTION}

In order to provide light sources across the wavelength of the visible spectrum, visible semiconductor lasers (VSLs) currently uses two material systems. Nitride based material systems $(\mathrm{InGaN} / \mathrm{GaN})$ are available for violet to green wavelengths ( $405-530 \mathrm{~nm})$ and phosphide based (InGaP/InAlGaP) cover the red spectrum (632-690nm). The greenyellow-orange range $(\sim 530-632 \mathrm{~nm})$ is not covered by any commercially VSLs. Therefore, there is a wavelength 'gap' in the visible color range. Covering the visible spectrum range is important for applications like solid state lighting, medicine, indicator lights, horticulture, displays, and in visible light communications (VLC). ${ }^{1,2}$ The full coverage of the visible spectrum with commercially viable VSLs would allow semiconductors solid state lighting system with very high color rendering index (CRI), a measure of well light sources reproduce the color of the objects in natural lighting, and

\footnotetext{
*boon.ooi@kaust.edu.saｈttps://photonics.kaust.edu.sa
} 
increase the capabilities of VLC systems including Li-Fi. ${ }^{2}$ Allowing multiple wavelengths to be used will increase VLC bandwidth and visible lasers with $\mathrm{GHz}$ modulation bandwidth can replace the slow modulation rate of LED technology. Ideally, the existing InGaP/InAlGaP or InGaN/GaN based material system should be used to cover this missing wavelength gap, as this would reduce the complexity and costs of producing such VSLs, but there are issues with extending the wavelength coverage of both.

For the InGaP/InAlGaP based system, increase the aluminum content and emission can reach the green-yellow-orange emission, but moving to shorter wavelength reduces the confinement of electrons and holes in the quantum wells, prohibiting the growth of high quality quantum well structures. ${ }^{3}$ Furthermore, the efficacy plummets due to tremendous hike in the density of the non-radiative centers. For the InGaN/GaN based system, all that is needed is to increase indium content to reach longer wavelength but, large strain and indium segregation prevent the growth of high quality light emitters in this color range. ${ }^{4}$ The only access to the orange-yellow-green range has been achieved by application of high external pressures and low-temperature ${ }^{5}$, frequency doubling of infrared lasers ${ }^{6}$, or by frequency doubling of diode pumped solid-state lasers. ${ }^{7}$ In this paper a novel strain-induced quantum well intermixing $(\mathrm{QWI})^{8}$ technique employed to promote interdiffusion via application of a thick dielectric encapsulant layer, cycles annealing at elevated temperature. With this QWI technique, we have successful tuned the bandgap of InGaP/InAlGaP structure from $\sim 640 \mathrm{~nm}$ to $565 \mathrm{~nm}$ $(\sim 250 \mathrm{meV})$ and demonstrated the first room temperature (RT) orange laser with a decent output power of $\sim 46 \mathrm{~mW}$ at $608 \mathrm{~nm}$ and yellow superluminescent diode (SLD) with a highest power of $\sim 4.5 \mathrm{~mW}$ on this material system. We believe our present yellow emitter will revolutionize laser eye surgery, since it is better absorbed by haemoglobin than the present green emitters. As this novel QWI technology is volume scalable and cost-effective it could be used for local cancer treatment, control cholesterol levels and redefine time.

\section{EXPERIMENT}

The single quantum well (SQW) InGaP/InAlGaP laser structure was grown on $10^{\circ}$ offcut GaAs substrate using metalorganic chemical vapor deposition (MOCVD) as shown in Fig.1. The structure is described in detail elsewhere. ${ }^{1}$. The emission of the laser was designed to be at $638 \pm 2 \mathrm{~nm}$. For the novel QWI process, we studied the effect of the thickness $(0.2-4 \mu \mathrm{m})$ of dielectric encapsulant (external strain), annealing temperature $\left(700-1000^{\circ} \mathrm{C}\right)$, annealing duration $(5-240 \mathrm{~s})$ and number of cycles of annealing to identify the optimal process conditions for preserving the surface morphology, photoluminescence (PL) characteristics and electrical properties. For the purpose of this work a $1 \mu \mathrm{m}$ thick $\mathrm{SiO}_{2}$ cap, $950^{\circ} \mathrm{C}$ annealing temperature, cycles annealing of 30s duration was applied to achieve the desired emission wavelength and optimal process conditions. The bandgap shifts induced by the above procedure were measured at RT using PL spectroscopy equipped with a $473 \mathrm{~nm}$ cobalt laser as the excitation source. Wafers were then processed using conventional processing and $1 \mathrm{~mm}$ long and $75 \mu \mathrm{m}$ wide ridge devices were used for optoelectronic characterization. All the devices were mounted on ceramic tiles and probed directly. The measurements were carried out at a tile temperature of $295 \mathrm{~K}$, while pulsed operation $(0.5 \mu$ s pulsed duration, $0.1 \%$ duty cycle) is used to minimize self-heating effects. 


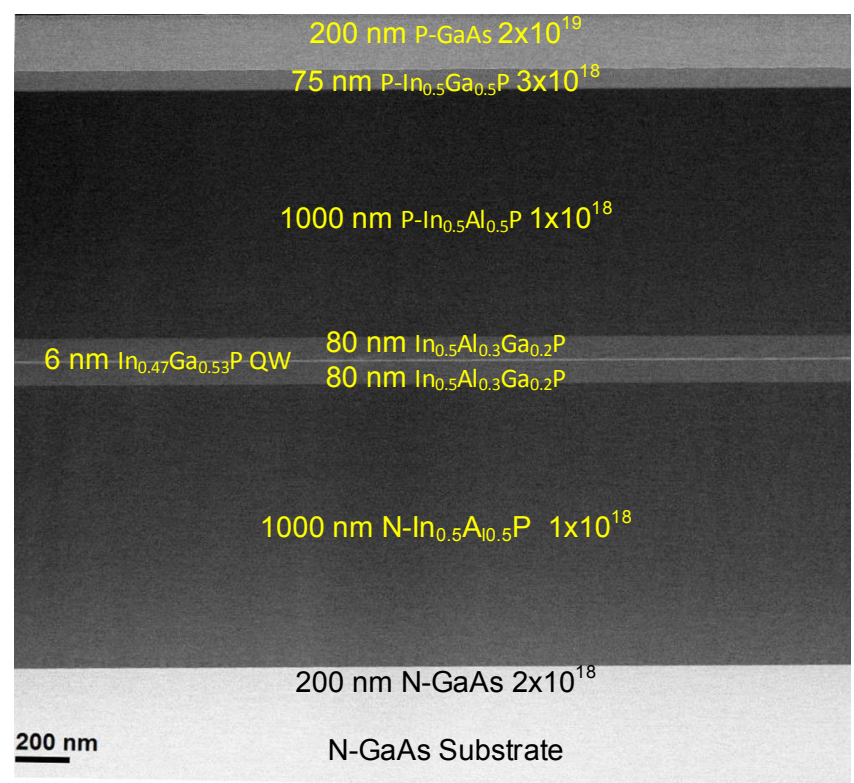

Figure 1. Dark field (002) cross-section TEM image of the InGaP/InAlGaP laser structure with an InGaP SQW.

\section{RESULTS AND DISCUSSION}

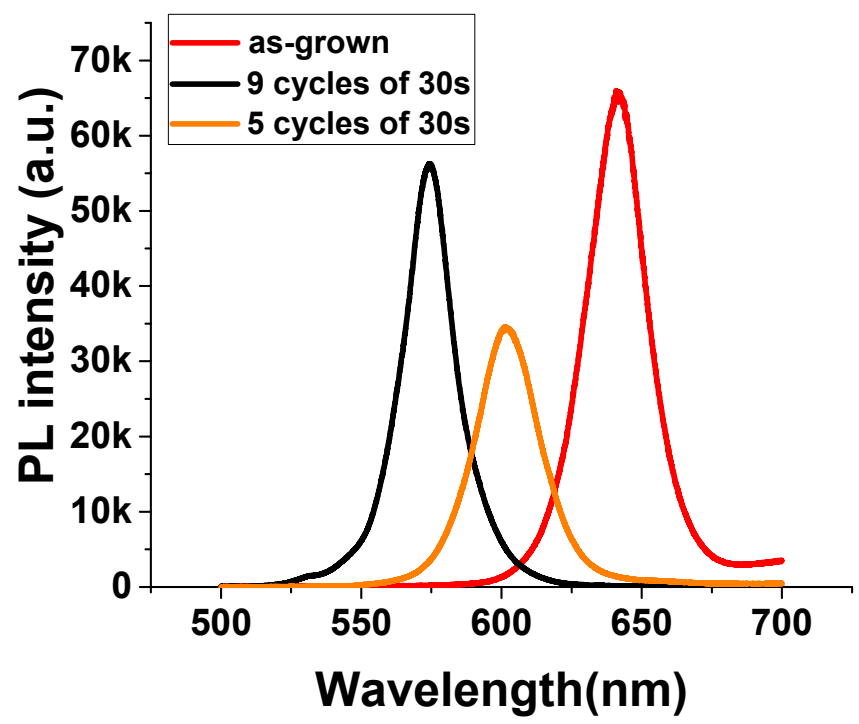

Figure 2. RT PL spectra of as-grown and novel QWI InGaP/InAlGaP sample after annealed at $950^{\circ} \mathrm{C}$ for 5 and 9 cycles of $30 \mathrm{~s}$ duration.

Fig. 2, shows RT PL spectra of as-grown and novel QWI InGaP /InAlGaP sample after annealing at $950^{\circ} \mathrm{C}$ for 5 and 9 cycles of 30s duration. A bandgap blueshift of 39nm $(\sim 125 \mathrm{meV})$ and $65 \mathrm{~nm}(\sim 220 \mathrm{meV})$ was observed for 5 and 9 cycles of 30s respectively. Similar bandgap blueshift is also observed for samples undergone annealing for multiple cycles 60 , 
90, 120, 180 and 240s but was not considered in this study due to drastic reduction in PL intensity whilst sample annealed for 5 cycles of $30 \mathrm{~s}(601 \mathrm{~nm})$ maintained high PL intensity with factor of $\sim 1.5$ reduction in PL intensity with negligible increase in full-width at half maximum (FWHM).

The 9 cycle annealed sample (575nm) shown negligible reduction in peak PL intensity, coupled with improved FWHM as compared to as-grown sample $(640 \mathrm{~nm})$, which is important for subsequent fabrication of laser devices. Fig. 3(a \& b) shows the representative TEM micrograph for the samples annealed at $950^{\circ} \mathrm{C}$ for 9 cycles of $30 \mathrm{~s}$ and as-grown sample. As can be seen from the figure that the composition at the interface of the quantum well (QW) $(6 \mathrm{~nm}-\mathrm{InGaP})$ and the barrier $(80 \mathrm{~nm}-\mathrm{InAlGaP})$ was abrupt like in the case of epitaxial growth samples. Infact HRTEM shown that the quantum well interface was sharper and smoother than noted in the as grown sample and that explains the improved FWHM of this sample. The results clearly suggest that our novel strain-induced QWI technique is highly efficient for interdiffusion of composite atoms $(\mathrm{Al} / \mathrm{Ga})$ between barriers and QW. By applying our novel QWI technique, we obtained bandgap shift of $\sim 75 \mathrm{~nm}(\sim 250 \mathrm{meV})$ after 15 cycles of annealing for $30 \mathrm{~s}$ with $\sim 6$ times reduction in PL intensity as compared to asgrown (not shown). This is the maximum bandgap shift in this material system at this short wavelength of $\sim 640 \mathrm{~nm}$.

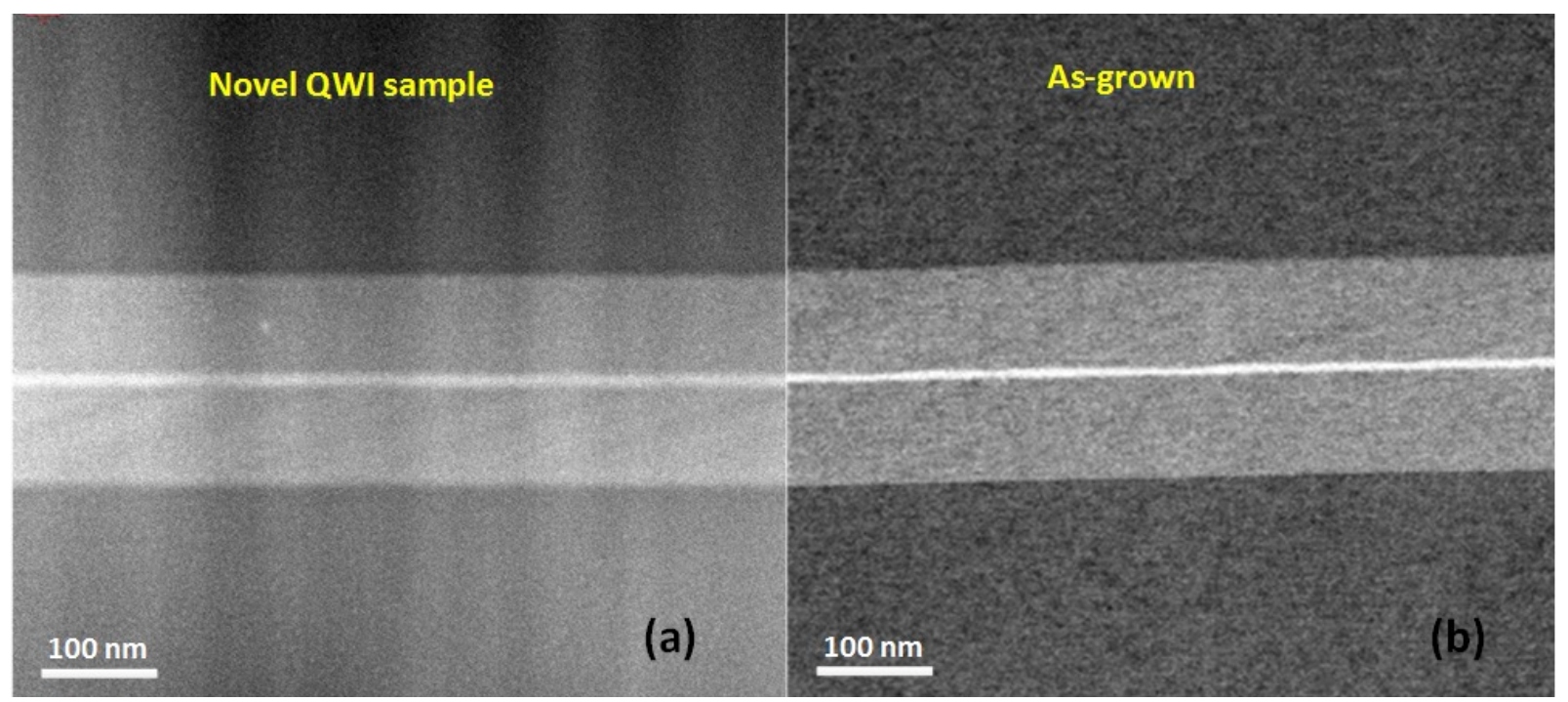

Figure 3. Dark field (002) cross-section TEM image of (a) novel QWI sample annealed at $950^{\circ} \mathrm{C}$ for 9 cycles of 30 s duration (b) asgrown sample.

We attribute reduction in peak PL intensity not only due to degradation of the material quality but also due to reduced conduction band offset and high carrier leakage. We would like to point out here that TEM images of the highly intermixed samples (PL @ 565nm) shows good QW and barrier interface with negligible increase in QW width. This achievement may enable the prospect of a powerful and simplified fabrication route for optoelectronic devices integration, and low optical loss photonic integrated circuits based on the InGaP/InAlGaP material system. 

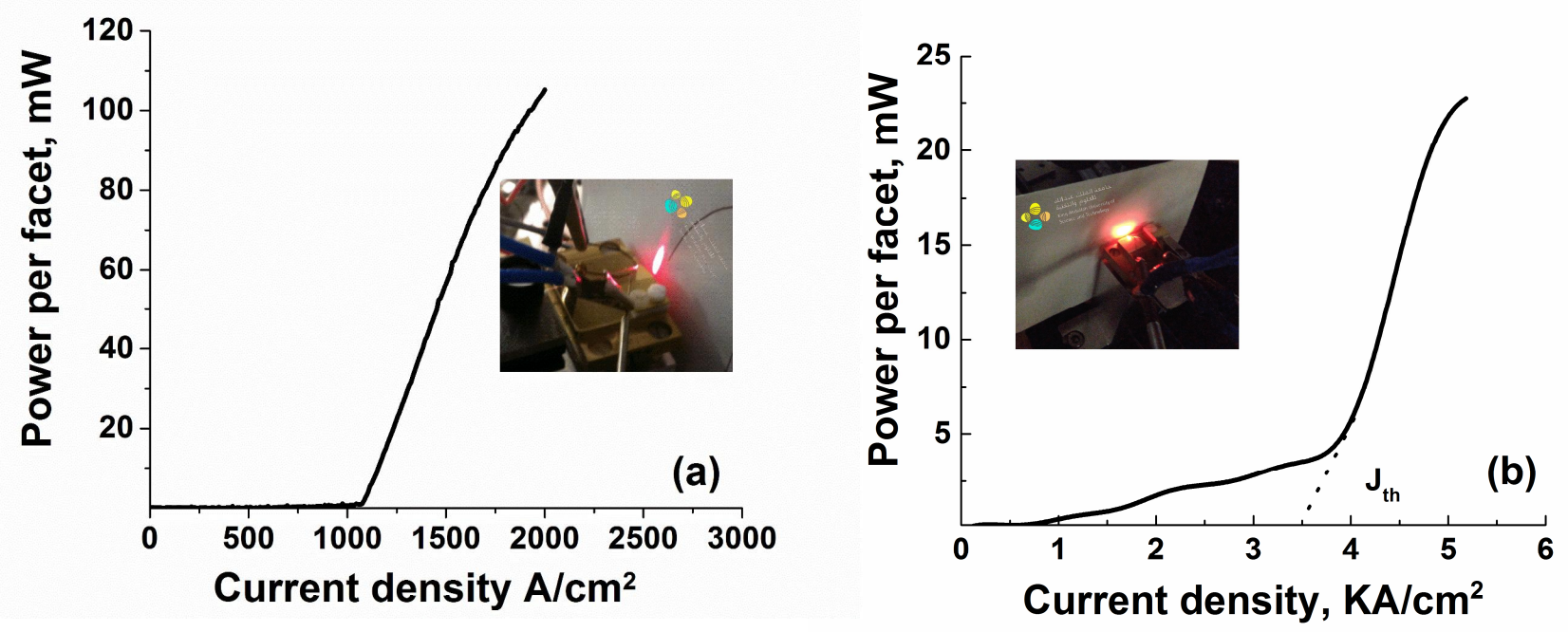

Figure 4. Power-current characteristics as a function of current density from $1 \mathrm{~mm}$ long and $75 \mu \mathrm{m}$ wide broad area laser fabricated from InGaP/InAlGaP (a) as grown and (b) 5 cycles of novel QWI samples. Inset: Red and orange lasing spot emitting at a current injection of $1.2 \mathrm{~J}_{\mathrm{th}}$.

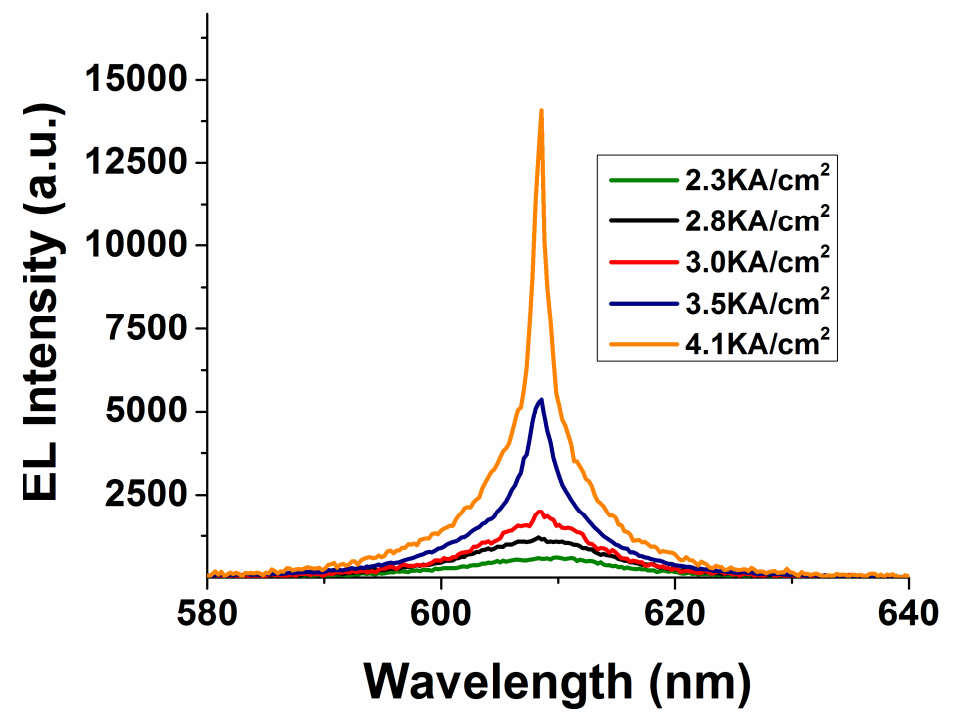

Figure 5. RT orange lasing spectra obtained as a function of current density from $1 \mathrm{~mm}$-long broad area laser fabricated from 5 cycles of novel QWI sample. 
Fig. 4 (a \& b) compares the optical power-current characteristics of a $1 \mathrm{~mm}$ long laser device fabricated from as-grown and from the 5 cycles of of novel QWI sample. The inset shows the red and orange lasing spot at 1.2 times $\mathrm{J}_{\mathrm{th}}$. Threshold current density $\left(\mathrm{J}_{\mathrm{th}}\right)$ of $3.4 \mathrm{kAcm}^{-2}$ and total output power of $\sim 46 \mathrm{~mW}$ (two facets) was noted for the for QWI sample whereas the as-grown sample of similar length exhibited $\sim 3$ times less current density and $\sim 6$ times more output power at the lasing wavelength of $\sim 640 \mathrm{~nm}$. The increased current density and decreases output power of the novel QWI sample is attributed to the increased optical losses due to the diffusion of aluminum in the $\mathrm{QW}$, strong and deleterious temperature effects due to the indirect minimum population and electron leakage due to smaller conduction band offset. Fig. 5, shows the RT orange lasing spectra obtained as a function of current density from $1 \mathrm{~mm}$-long broad area laser fabricated from 5 cycles of novel QWI sample. As compared to the PL in Fig. 2, the lasing emission red-shifted by $\sim 7 \mathrm{~nm}$ as compared to PL emission. This is due to the leakage of injected current, particularly from the active region into the p-type cladding owing to the smaller conduction band offset.
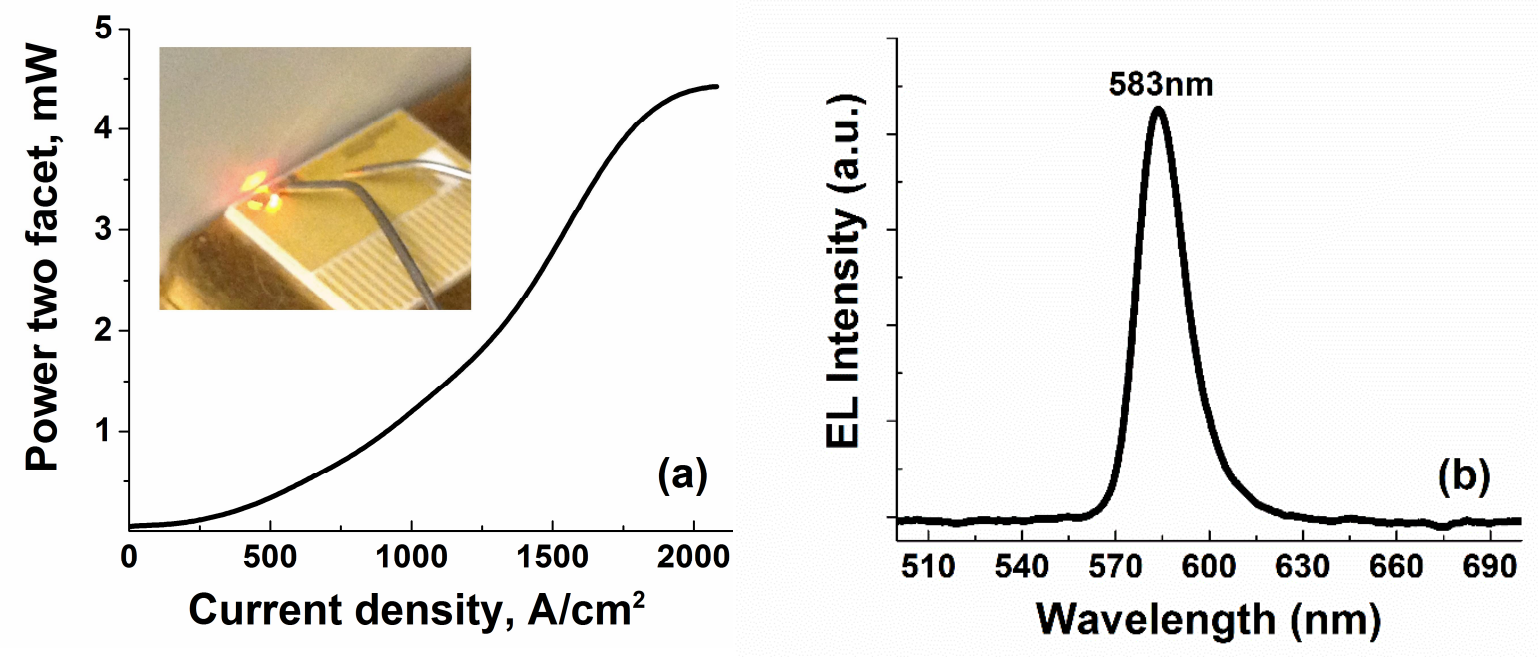

Figure 6. (a) Power-current characteristics as a function of current density from $1.5 \mathrm{~mm}$ long and $75 \mu \mathrm{m}$ wide broad area laser fabricated from InGaP/InAlGaP inter-diffused sample. Inset: yellow amplified spontaneous emission spot at 583nm, (b) amplified spontaneous emission spectra at $583 \mathrm{~nm}$.

Fig. 6 (a) shows optical power-current characteristics of a $1.5 \mathrm{~mm}$ long and $75 \mu \mathrm{m}$ wide laser device at RT fabricated from 9 cycles of novel QWI (sample shown in fig.2). Inset shows the yellow amplified spontaneous spot. Fig 6 (b) shows the $583 \mathrm{~nm}$ amplified spontaneous emission spectra at a current density of $150 \mathrm{~s} 0 \mathrm{~A} / \mathrm{cm}^{2}$. The laser devices exhibit superlinear increase in optical power with increasing current density. We noted $4.5 \mathrm{~mW}$ total output power at a current density of $1750 \mathrm{~A} / \mathrm{cm}^{2}$. This is the highest reported power at this wavelength on this material system at RT. The second best reported power was $0.36 \mu \mathrm{W}$ (two facets) on the strained-InGaP quantum well heterostructure grown on a transparent, compositionally graded AlInGaP buffer. ${ }^{9}$ As a comparison to the orange laser, yellow emitting device was $50 \%$ longer but the power decreased by a factor of $\sim 10$ for only $100 \mathrm{meV}$ conduction band offset between both the devices. The increased current density and decreases output power is attributed to the increased optical losses due to the diffusion of 
aluminium in the QW which was confirmed by a series of Electron Energy Loss Spectroscopy (EELS) combined with HRTEM (not shown), strong and deleterious temperature effects due to the indirect minimum population and electron leakage due to smaller conduction band offset between InAlGaP and InAlP. The results are very encouraging given that the cladding has no complicated multiquantum barrier (MQB) to suppress the carrier overflow. Probably MQB will allow lower threshold and high output power and bring us one step closer to demonstrating yellow semiconductor lasers.

\section{CONCLUSION}

We present the visible semiconductor emitters using novel strain induced quantum well intermixing technique on InGaP/InAlGaP red laser structure. A maximum bandgap shift of $\sim 250 \mathrm{meV}$ with original wavelength of $640 \mathrm{~nm}$ is observed in this material system. Orange laser and yellow emitter with the peak wavelength at $608 \mathrm{~nm}$ and $583 \mathrm{~nm}$ with relatively good performance have been demonstrated at room temperature.

\section{ACKNOWLEDGEMENTS}

The authors gratefully acknowledge the financial support from KAUST baseline funding, Competitive Research Grant (CRG), and KACST Technology Innovation Center for Solid State Lighting at KAUST.

\section{REFERENCES}

[1] Majid, M.A., Al-Jabr, A.A., Oubei, H.M., Alias, M.S., Anjum, D.H., Ng, T.K. and Ooi, B.S., "First demonstration of InGaP/InAlGaP based orange laser emitting at $608 \mathrm{~nm}$," Electronics Letters, 51(14), 1102$1104(2015)$

[2] Janjua, B., Oubei, H.M., Retamal, J. R. D., Ng, T. K., Tsai, C.T., Wang, H.Y., et al., "Going beyond 4 Gbps data rate by employing RGB laser diodes for visible light communication," Optics express, 23, 18746-18753 (2015).

[3] Nakamura, S., Senoh, M., Iwasa, N., and Nagahama, S.I., "High-brightness InGaN blue, green and yellow lightemitting diodes with quantum well structures," Japanese Journal of Applied Physics, part 2 letters, 34, 797 (1995).

[4] Kondo, M., Okada, N., Domen, K., Sugiura, K., Anayama, C., and Tanahashi, T., "Origin of nonradiative recombination centers in AlGaInP grown by metalorganic vapor phase epitaxy," Journal of electronic materials, 23, 355-358 (1994).

[5] Bohdan, R., Bercha, A., Trzeciakowski, W., Dybała, F., Piechal, B., Sanayeh, M.B., Reufer, M., Brick, P.: "Yellow AlGaInP/InGaP laser diodes achieved by pressure and temperature tuning," Journal of Applied Physics, 104 (6), 063105-063105-5 (2008).

[6] Lee, J. H., Lee, S. M., Kim, T., and Park, Y. J., "7 W high-efficiency continuous-wave green light generation by intracavity frequency doubling of an end-pumped vertical external-cavity surface emitting semiconductor laser", Appl. Phys. Lett., 89(24), 241107 -241107-3 (2006).

[7] Miller, G. D., Batchko, R. G., Tulloch, W. M., Weise, D. R., Fejer, M. M. and Byer, R. L.: "42\%-efficient single-pass cw second-harmonic generation in periodically poled lithium niobate," Opt. Lett., 22(24), 18341836 (1997).

[8] Ooi, B. S., McIvlaney, K., Street, M. W., Saher Helmy, A., Ayling, S. G., Bryce, A. C., and J. H. Marsh, "Selective quantum well intermixing in GaAs/AlGaAs structures using impurity free vacancy diffusion", IEEE J. Quantum Electron, 33, 1784 -1793 (1997).

[9] McGill, L.M, Wu, J. and Fitzgerald, E. A., "Yellow-Green emission for ETS-LEDs and lasers based on a strained InGaP quantum well heterostructure grown on a transparent, compositionally graded AlInGaP buffer," Mat. Res. Soc. Symp. Proc., 744 (2003) 